\title{
New data on the morphology of Procamallanus (Procamallanus) annulatus and Procamallanus (Spirocamallanus) monotaxis (Nematoda: Camallanidae) from marine fishes off New Caledonia
}

\author{
F. MORAVEC ${ }^{1}$, J.-L. JUSTINE ${ }^{2,3}$
}

\begin{abstract}
${ }^{1}$ Institute of Parasitology, Biology Centre of the Academy of Sciences of the Czech Republic, Branišovská 31, 37005 České Budějovice, Czech Republic, E-mail: moravec@paru.cas.cz; ${ }^{2}$ UMR 7138 Systématique, Adaptation, Évolution, Muséum National d'Histoire Naturelle, Case postale 52, 57, rue Cuvier, 75231 Paris cedex 05, France; ${ }^{3}$ Aquarium des Lagons, B. P. 8185, 98807 Nouméa, Nouvelle-Calédonie
\end{abstract}

\begin{abstract}
Summary
Two little-known nematode species of the family Camallanidae, intestinal parasites of marine perciform fishes, are reported from off New Caledonia: Procamallanus (Procamallanus) annulatus Yamaguti, 1955 from the goldenlined spinefoot Siganus lineatus (Valenciennes) (Siganidae) and Procamallanus (Spirocamallanus) monotaxis (Olsen, 1952) from the longspine emperor Lethrinus genivittatus Valenciennes and the slender emperor Lethrinus variegatus Valenciennes (both Lethrinidae). Detailed light and electron microscopical studies (the latter used for the first time in these species) revealed some taxonomically important, previously not observed features, such as the presence of deirids, six crescent-shaped elevations surrounding the mouth, three poorly developed protuberances on the female tail tip and circumcloacal papillae and phasmids on the male tail in P. annulatus, and the presence of a circumoral flange, only 12 (instead of 14 reported) cephalic papillae, the shape of deirids and similar numbers $(14-17$ and $14-15)$ of spiral ridges in the male and female buccal capsules in $P$. monotaxis. The fourth-stage larva of $P$. annulatus is described for the first time. Firststage larvae of both species were found to possess several digital processes at the tail tip. The present findings represent new host and geographical records of these parasite species.
\end{abstract}

Keywords: parasitic nematode; Procamallanus (Procamallanus); Procamallanus (Spirocamallanus); marine fish; Siganus; Lethrinus; New Caledonia; South Pacific Ocean

\section{Introduction}

Nematodes of the family Camallanidae seem to be frequent parasites of marine fishes in the South Pacific Ocean (Lester \& Sewell, 1989; Rigby \& Adamson, 1997; Rigby \& Font, 1997; Rigby et al., 1997, 1998, 1999; Moravec et al., 2006a), but their species composition in this region remains insufficiently known. Only three nominal species of adult camallanids have so far been reported from marine fishes in New Caledonian waters: Camallanus carangis Olsen, 1954 from Nemipterus furcosus (Valenciennes) (Nemipteridae), Parupeneus ciliatus (Lacépède) and Upeneus vittatus (Forsskål) (both Mullidae); Procamallanus (Spirocamallanus) guttatusi (Andrade-Salas, PinedaLópez et García-Magaña, 1994) (reported under the synonym Spirocamallanus philippinensis Machida et Taki, 1985) from Digramma pictum (Thunberg) (Haemulidae), and Procamallanus (Spirocamallanus) variolae Moravec, Justine et Rigby, 2006 from Variola albimarginata Baissac and Epinephelus fasciatus (Forsskål) (both Serranidae) (Baillon, 1991; Moravec et al., 2006a; Justine, 2010); however, in addition, Baillon (1991) recorded one unidentified Camallanus sp. from D. pictum, Moravec et al. (2006a) reported three unidentified Procamallanus (Spirocamallanus) spp. (only females available) from other fishes, and Sigura and Justine (2008) reported Procamallanus sp. from Epinephelus cyanopodus (Richardson). Two other species of camallanids, Camallanus cotti Fujita, 1927 and Procamallanus (Procamallanus) pacificus Moravec, Justine, Würtz, Taraschewski et Sasal, 2006 were reported from New Caledonian freshwater fishes (Moravec \& Justine, 2006; Moravec et al., 2006b).

Parasitological examinations of marine fishes off the coast of New Caledonia yielded, among other helminths, two intestinal camallanid species of Procamallanus Baylis, 1923 parasitizing the golden-lined spinefoot Siganus lineatus (Valenciennes) (Siganidae), the longspine emperor Lethrinus genivittatus Valenciennes and the slender emperor Lethrinus variegatus Valenciennes (both Lethrinidae), that were not previously reported from this region. Their detailed light microscopy (LM) and scanning electron microscopy (SEM) examination made it possible to find some new, taxonomically important features of these hitherto little-known nematode species and to redescribe 
them. Results of this study are presented herein.

Siganus lineatus (maximum body length $43 \mathrm{~cm}$ ), Lethrinus genivittatus (up to $25 \mathrm{~cm}$ ) and L. variegatus (up to $20 \mathrm{~cm}$ ) are tropical, reef-associated marine fishes with Indo-West Pacific distributions (Froese \& Pauly, 2010).

\section{Materials and methods}

Fish were collected by fishing with hook and line (Lethrinus spp.), or by spear-fishing and from the fish market of Nouméa (Siganus spp.). All fish were measured, weighted and photographed, and assigned an unique registration number (JNC). The nematodes for morphological studies were fixed in hot $4 \%$ formaldehyde solution, sometimes in $70 \%$ ethanol. For light microscopical examination, they were cleared with glycerine. Drawings were made with the aid of a Zeiss microscope drawing attachment. Specimens used for scanning electron microscopy were postfixed in $1 \%$ osmium tetroxide, dehydrated through a graded acetone series, critical point dried and sputter-coated with gold; they were examined using a JEOL JSM-7401F scanning electron microscope at an accelerating voltage of 4 $\mathrm{kV}$ GB low. All measurements are in micrometres unless otherwise stated. Fish names follow FishBase (Froese \& Pauly, 2010).

\section{Results}

Family Camallanidae Railliet et Henry, 1915

Procamallanus (Procamallanus) annulatus Yamaguti,
1955 (Figs. 1, 2)

\section{Description}

General: Medium-sized nematodes with finely transversely striated cuticle (Fig. 2A, D). Body of live specimens reddish, of fixed specimens light grey to brown. Mouth aperture circular, surrounded by six flat, crescent-shaped elevations and four submedian cephalic papillae (Figs. 1C, 2A, C). Pair of small lateral amphids present (Figs. 1C, 2A, B). Buccal capsule orange-brown, thick-walled, barrelshaped, slightly longer than wide, with well developed basal ring; basal, narrowed part of buccal capsule proper anterior to basal ring somewhat thickened, forming conspicuous circular ledge. Inner surface of capsule smooth, without any spiral ridges (Fig. 1A, B). Muscular oesophagus somewhat shorter than glandular oesophagus; both parts of oesophagus slightly expanded near their posterior ends (Fig. 1A). Intestine brown, narrow. Deirids small, simple, with rounded end, situated some distance anterior to level of nerve ring or, sometimes, at about mid-way between posterior end of buccal capsule and nerve ring (Figs. 1A, B, I, 2D). Excretory pore somewhat posterior to junction of both parts of oesophagus (Fig. 1A). Tail of both sexes conical, with blunt tip.

Male (6 specimens; measurements of 1 additional, juvenile specimen in parentheses): Length of body 16.99-19.94 (8.96) mm, maximum width 367 - 490 (231). Buccal cap- sule including basal ring 117 - 129 (99) long, its width 99 - 108 (93); basal ring $12-18$ (12) long and $63-69$ (66) wide; circular ledge 15 - 18 (9) high. Maximum width/length ratio of buccal capsule $1: 1.14-1.30$ (1:1.06). Length of muscular oesophagus 408-490 (408), maximum width $69-90$ (60); length of glandular oesophagus $530-$ 680 (490), maximum width $63-78$ (63); length ratio of muscular and glandular oesophagus 1:1.3 - 1.5 (1:1.2). Length of entire oesophagus and buccal capsule representing $6-7$ (11) \% of body length. Nerve ring, deirids and excretory pore $272-354$ (258), $228-270$ (270) and 558 - 734 (490), respectively, from anterior extremity. Posterior end of body ventrally bent, provided with wide, vesiculated caudal alae supported by pedunculate papillae; alae anteriorly interconnected by mound, forming thus a kind of pseudosucker, and posteriorly reaching to tail tip (Figs. 1D, 2E, F). Preanal papillae: 3 pairs of equally spaced subventral pedunculate papillae; postanal papillae: 5 pairs of anterior subventral papillae and 1 pair of posterior lateral phasmids; additional 2 pairs of small transversely elongate sessile ventral papillae surrounding cloacal opening (Fig. 1D, J, K). Spicules similar in shape, unequal, with pointed distal ends; large (right) spicule 321 - 387 (285) long, with narrowed, ventrally bent, alate distal tip; small (left) spicule somewhat less sclerotized, 231 299 (201) long. Length ratio of spicules 1:1.07 - 1.47 (1:1.42). Gubernaculum well sclerotized, conspicuous, 135 - 165 (138) long (Fig. 1D, J, K). Tail conical, 153 - 300 (190) long, with rounded tip.

Female ( 5 gravid specimens; measurements of 2 subgravid specimens in parentheses): Length of body $37.97-46.76$ (33.05 - 42.12) mm, maximum width 707 - 938 (680 802). Buccal capsule including basal ring 138 - 153 (150 $153)$ long and $126-132(120-132)$ wide; basal ring 15 $21(15-21)$ long and $84-99(84-87)$ wide; circular ledge 15 - 18 (15 - 18) high. Maximum width/length ratio of buccal capsule 1:1.10 - 1.21 (1:1.21 - 1.25). Length of muscular oesophagus 517 - 639 (476 - 544), maximum width $102-122$ (93); length of glandular oesophagus 707 - 911 (721 - 748), maximum width 81 - 122 (81); length ratio of muscular and glandular oesophagus 1:1.3 - 1.5 (1:1.4 - 1.5). Length of entire oesophagus and buccal capsule representing $3-4(3-4) \%$ of body length. Nerve ring, deirids and excretory pore $326-449$ (340 - 381), 282 $-300(300-306)$ and $748-1142$ (748), respectively, from anterior extremity. Vulva pre-equatorial, $15.40-17.50$ $(13.10-1485) \mathrm{mm}$ from anterior extremity, at $36-41(35$ - 40) \% of body length. Anterior vulval lip somewhat elevated. Vagina muscular, directed posteriorly from vulva. Uterus filled with larvae $555-609$ (-) long and $21-$ 24 (24) wide; oesophagus 120 - 132 long $(20-22 \%$ of body length), tail 111 - 126 long (19 - $22 \%$ of body length); tail tip of larva bearing several minute digit-like processes (Fig. 1G, H). Tail of female conical, $408-558$ (367 - 435) long; tail tip blunt, with three slightly outlined protuberances (Fig. 1L, M).

Female fourth-stage larva (1 specimen): Body whitish, $7.93 \mathrm{~mm}$ long, maximum width 245. Buccal capsule co- 
lourless, 84 long including basal ring, and 84 wide; basal ring 9 long and 45 wide; circular ledge slightly outlined, 12 high (Fig. 1E). Maximum width/length ratio of buccal capsule 1:1. Length of muscular oesophagus 367, maximum width 60 ; length of glandular oesophagus 408 , maximum width 78; length ratio of muscular and glandular oesophagus 1:1.1. Length of entire oesophagus and buccal capsule representing $11 \%$ of body length. Nerve ring, deirids and excretory pore 225,171 and 571, respectively, from anterior extremity; deirids conical, pointed. Vulva pre-equatorial, $2.45 \mathrm{~mm}$ from anterior extremity (at $36-$ 41 (31\% of body length), still covered by cuticle. Uterus

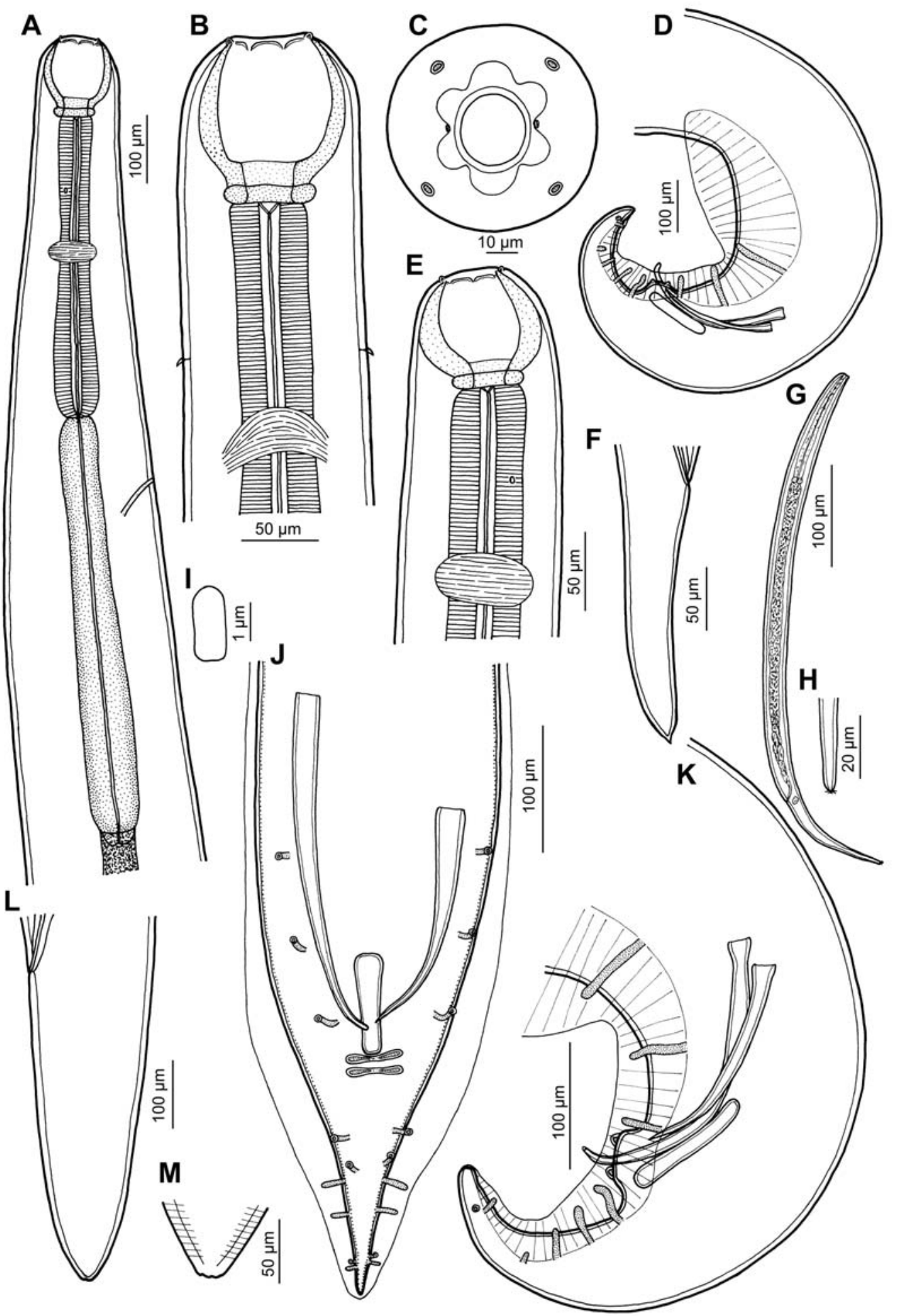

Fig. 1. Procamallanus (Procamallanus) annulatus Yamaguti 1955. A, B, C) Anterior end of male, lateral, dorsoventral and apical views, respectively; D) Posterior end of male, lateral view; E) Anterior end of fourth-stage larva, lateral view; F) Tail of fourth-stage larva, lateral view; G) First-stage larva from uterus; H) Tail tip of first-stage larva; I) Shape of deirid, lateral view; J, K) Tail of male, ventral and lateral views; L) Tail of gravid female, lateral view; M) Tail tip of gravid female. 
tubular, empty. Tail conical, 204 long, sharply pointed at tip (Fig. 1F).

Host: Golden-lined spinefoot, Siganus lineatus (Siganidae, Perciformes).

Site of infection: Intestine and pyloric caeca.

Locality: New Caledonia (off Nouméa; Nouméa fish market).

Prevalence and intensity: 4 fish infected/ 6 fish examined; 1 -61 (mean 18) specimens per fish.
Deposition of voucher specimens: Muséum National d'Histoire Naturelle, Paris (JNC 1992, 2860, 2873, 2998); Institute of Parasitology, BC ASCR, České Budějovice (N-953).

Comments: The general morphology of specimens of the present material, in particular the presence of the buccal capsule without spiral ridges or other ornamentations on inner surface, shows clearly that they belong to the nominotypical subgenus of Procamallanus Baylis, 1923. Of

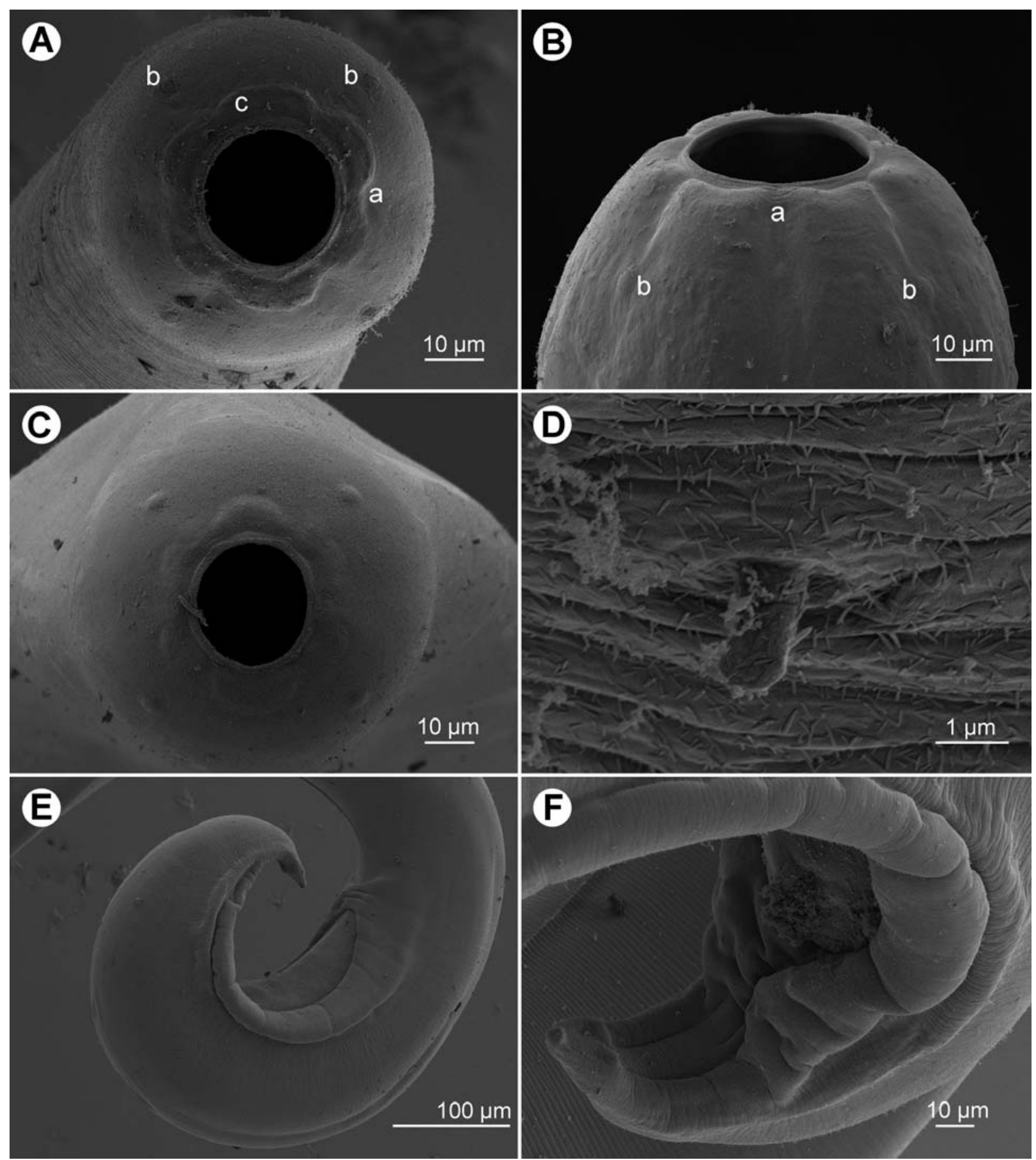

Fig. 2. Procamallanus (Procamallanus) annulatus Yamaguti 1955, SEM micrographs. A, B) Cephalic end of female, apical and lateral views; C) Cephalic end of male, apical view; D) Deirid; E) Posterior end of male showing sucker-like formation formed by caudal alae, sublateral view; F) Posterior end of male tail, ventrolateral view. a - amphid, b - cephalic papilla, c - circumoral sclerotized elevation. 
several species of this subgenus described from marine fishes (see Ivashkin et al., 1971; Petter, 1979; Arya, 1984), the most similar are the following four species, all described from Siganus spp. of the Indo-Pacific region: $P$. sigani Yamaguti, 1935 from S. fuscescens (Houttuyn) from Japan (Inland Sea of Japan and Pacific coast of Mie and Wakayama Prefectures) (Yamaguti, 1935) and later reported from S. guttatus (Bloch) and S. oramin (Bloch et Schneider) from North Borneo, Malaysia (Myer \& Kuntz, 1969); P. lonis Yamaguti, 1941 from S. unimaculatus (Evermann et Seale) from Okinawa, Japan (Yamaguti, 1941) and later reported from Australia from S. lineatus (Valenciennes) and S. punctatus (Schneider et Forster) from the Great Barrier Reef (Lester and Sewell, 1989) and from Platax batavianus Cuvier (Ephippidae, Perciformes) off the coast of Queensland (Smales, 2000); P. annulatus Yamaguti, 1955 from Siganus sp. from Celebes (Macassar), Indonesia, later also reported from S. stellatus (Forsskål) from Sri Lanka (Colombo) (Parukhin, 1971, 1976); and P. elatensis Fusco et Overstreet, 1979 from S. luridus (Rüppel) and S. rivulatus (Forsskål) from the Red Sea (Fusco \& Overstreet, 1979).

However, the original descriptions of all these four species are very incomplete, their measurements often overlap, their type specimens (except for P. elatensis) are in poor condition preventing their re-examination, so that it cannot be excluded that in fact they represent only one species. However, until their redescriptions based on newly collected topotypic materials are available, they should be considered valid. Of them, $P$. sigani, $P$. lonis and $P$. elatensis differ from New Caledonian specimens by distinctly shorter body not exceeding $16 \mathrm{~mm}$ in males and $28 \mathrm{~mm}$ in gravid females (vs. $17-20$ and $38-47 \mathrm{~mm}$ ); moreover, no gubernaculum and ring-like internal thickening (ledge) of the buccal capsule are mentioned to be present in $P$. sigani and $P$. lonis (but the latter is illustrated in $P$. lonis). Both the gubernaculum and the circular ledge are reported for $P$. elatensis, but the length ratio of spicules in this species is different $(1: 2.4-3.3$ vs. $1: 1.1-1.5)$ and the gubernaculum is V-shaped, weakly sclerotized, only $41-63$ long (vs. non-bifurcated, strongly sclerotized, 135 - 165 long); data on the location of the excretory pore anterior to the level of nerve ring and the absence of deirids in $P$. elatensis are evidently erroneous.

Body lengths (male $10-20 \mathrm{~mm}$, female $28-43$ ) and other measurements of $P$. annulatus are similar to those in the present specimens, both forms possess the buccal capsule with a distinct circular ledge and the gubernaculum of a similar shape and length, the number and distribution of male genital papillae are identical, and the lengths of larvae from uterus are also similar. Therefore, the New Caledonian specimens are considered to belong to $P$. $(P$.) annulatus, even though it was originally described from a different geographical region (Indonesia).

In the original description of P. annulatus, Yamaguti (1955) did not report some taxonomically important features, such as the presence of deirids, six crescent-shaped elevations surrounding the mouth, three poorly developed protuberan- ces on the female tail tip, and circumcloacal papillae and phasmids on the male tail. It is necessary to remark, however, that some of these features are difficult to observe by LM and can be properly studied only by SEM.

The fourth-stage larva of $P$. annulatus is described here for the first time; its buccal capsule resembles that in adults, including the presence of a weakly sclerotized circular ledge, but the deirids are pointed, as also the tail tip. It is remarkable that the tail tip of first-stage larvae of $P$. annulatus from uterus is provided with several digit-like processes. Similar caudal processes in first-stage larvae have hitherto been reported only for two species of the camallanid genus Camallanus Railliet et Henry 1915 (see Moravec and Justine, 2006) and recently also for the African species $P$. laeviconchus (Wedl, 1862) (Mašová et al., 2010).

It has been mentioned above that $P$. annulatus was previously reported from off Indonesia and Sri Lanka (Yamaguti, 1955; Parukhin, 1971, 1976). Its occurrence in New Caledonia, representing new host and geographical records, is not surprising, because many Siganus spp. have broad Indo-Pacific distributions. By sucking the host's blood, camallanids may be rather pathogenic and their heavy infections may be even a cause of death in cultured fish, as was documented, for example, for P. (S.) guttatusi (Andrade-Salas, Pineda-López et García-Magaña, 1994) in the reared orange-spotted spinefoot, Siganus guttatus, in a fish farm in the Philippines (Machida \& Taki, 1985; Moravec et al., 2004). Therefore, $P$. annulatus may also be a potential pathogen for cultured Siganus spp. This is of possible economic importance for rabbitfish aquaculture, which is presently under development in the Pacific and especially in New Caledonia (Teitelbaum et al., 2008).

\section{Procamallanus (Spirocamallanus) monotaxis (Olsen,} 1952) (Figs. 3, 4)

\section{Description}

General (based on specimens from L. genivittatus): Medium-sized nematodes with finely transversely striated cuticle. Mouth aperture oval to square (Figs. 3D, 4A, B), provided with narrow circumoral flange (Fig. 4A - D) and surrounded by six flat, crescent-shaped elevations and 12 submedian cephalic papillae arranged in three circles, each formed by four papillae; papillae of external circle distinctly larger. Pair of small lateral amphids present (Figs. 3D, 4A - D). Buccal capsule orange-brown, thick-walled, barrel-shaped, slightly longer than wide, with simple, well developed basal ring. Maximum width/length ratio of buccal capsule $1.1 .10-1.41$. Inner surface of capsule provided with $14-17$ spiral ridges in lateral view, $1-3$ of them being incomplete (Fig. 3A - C). Muscular oesophagus somewhat shorter than glandular oesophagus; both parts of oesophagus slightly expanded near their posterior ends (Fig. 3A). Intestine brown, narrow. Deirids small, simple, with rounded end situated at about mid-way between posterior end of buccal capsule and nerve ring (Figs. 3B, E, F, $4 \mathrm{G})$. Excretory pore somewhat anterior to junction of both 
parts of oesophagus (Fig. 3A). Tail of both sexes with two (dorsal and ventral) terminal cuticular spikes.

Male (7 specimens): Length of body $10.54-17.699 \mathrm{~mm}$, maximum width $218-354$. Buccal capsule including basal ring $87-93$ long, its width $66-69$; basal ring $9-12$ long and $42-54$ wide. Maximum width/length ratio of buccal capsule 1:1.26 - 1.41. Spiral ridges $14-17$, of which $1-3$ incomplete. Length of muscular oesophagus $367-490$, maximum width $66-109$; length of glandular oesophagus $517-721$, maximum width $78-122$; length ratio of muscular and glandular oesophagus 1:1.2 - 1.4. Length of entire oesophagus and buccal capsule representing $7-9 \%$
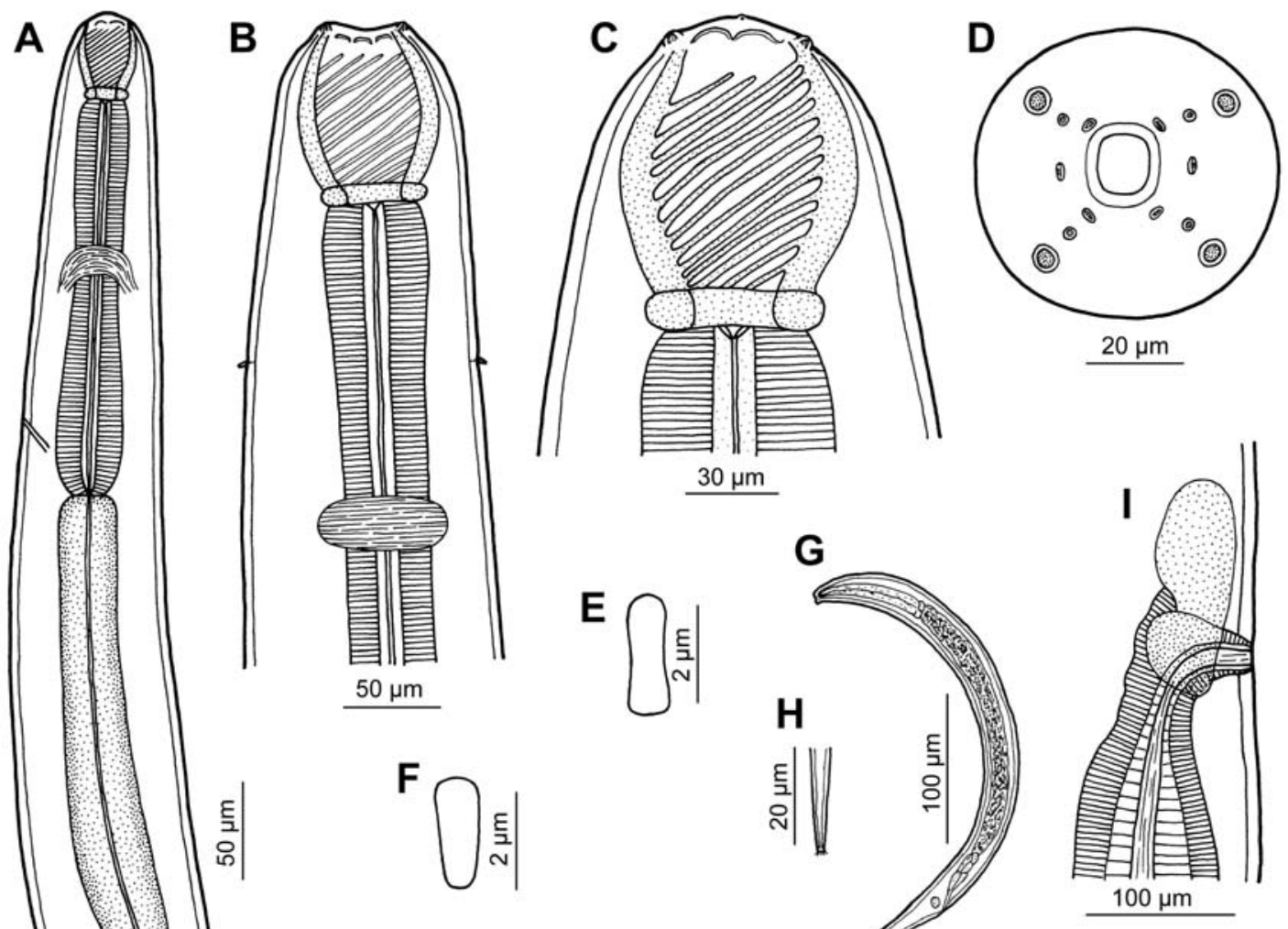

K
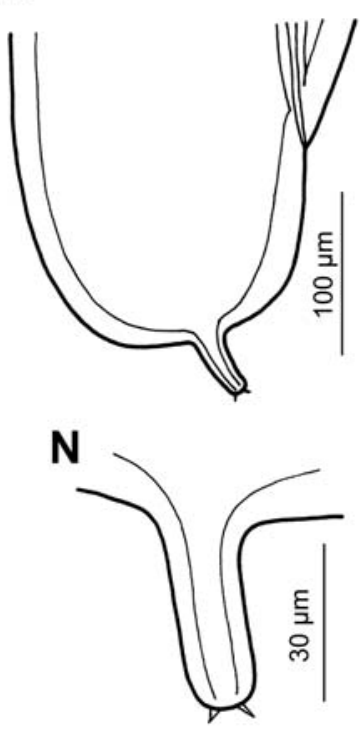

Fig. 3. Procamallanus (Spirocamallanus) monotaxis (Olsen 1952). A, B) Anterior end of male, lateral and dorsoventral views; C, D) Cephalic end of male, lateral and apical views; E, F) Shapes of deirids in two different specimens, lateral views; G) First-stage larva from uterus; H) Tail tip of first-stage larva; I) Vulva, lateral view; J) Tail of male, ventral view; K) Tail of gravid female, lateral view; L) Posterior end of male, lateral view; M) Right and left spicules, ventral view; N) Female caudal projection, lateral view. 
of body length. Nerve ring, deirids and excretory pore 286 - 313, 168 - 186 and 471 - 571, respectively, from anterior extremity. Posterior end of body ventrally bent, provided with wide, vesiculated caudal alae supported by pedunculate papillae; alae anteriorly interconnected by mound, forming thus a kind of pseudosucker, and posteriorly reaching to base of caudal terminal spikes (Figs. 3J, L, 4E). Preanal papillae: 3 pairs of equally spaced subventral pedunculate papillae; postanal papillae: 4 pairs of anterior subventral and 2 pairs of posterior lateral pedunculate papillae (last pair probably representing phasmids); additional 2 pairs of small transversely elongate sessile ventral papillae surrounding cloacal opening (Fig. 3J, L). Spicules similar in shape, unequal, with sharply pointed distal ends (Fig. 3L, M); large (right) spicule $279-$ 315 long; small (left) spicule less sclerotized, $171-213$ long. Length ratio of spicules 1:1.48 - 1.67. Gubernaculum not observed. Tail conical, $162-210$ long (Figs. 3J, L); length of terminal spikes $4-6$.

Female (5 gravid specimens; measurements of 1 subgravid
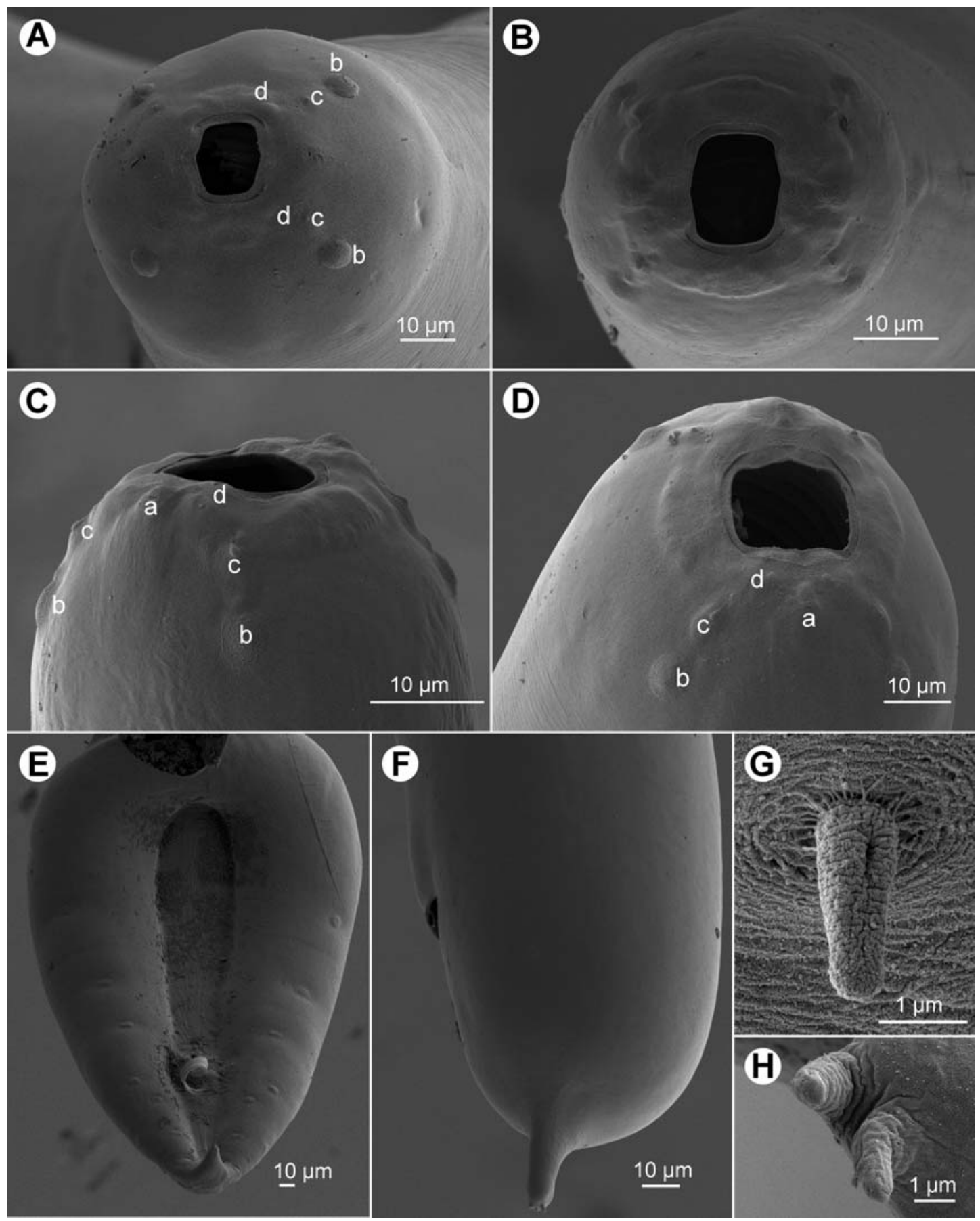

Fig. 4. Procamallanus (Spirocamallanus) monotaxis (Olsen, 1952), SEM micrographs. A) Cephalic end of male, apical view; B) Cephalic end of female, apical view; C, D) Cephalic end of female, sublateral and lateral views; E) Caudal end of male illustrating structure and shape of caudal alae, ventral view; F) Tail of female, lateral view; G) Deirid; H) Two spikes on distal tip of digit-like projection of female tail, sublateral view. a - amphid, b - cephalic papilla of most external circle, $c$ - cephalic papilla of middle circle, $d$ - cephalic papilla of most interior circle. 
specimen in parentheses): Length of body 24.64 (19.20) mm, maximum width 653 - 707 (476). Buccal capsule including basal ring 96 - 105 (105) long and $81-87$ (78) wide; basal ring 12 (12) long and 51 - 57 (51) wide. Number of spiral ridges $14-15$ (15), of which 2 (2) incomplete. Length of muscular oesophagus 571 - 612 (544), maximum width $120-123$ (105); length of glandular oesophagus 775 - 925 (762), maximum width 129 - 162 (105); length ratio of muscular and glandular oesophagus $1: 1.3$ - $1.6(1: 1.4)$. Length of entire oesophagus and buccal capsule representing 6 (7) \% of body length. Nerve ring, deirids and excretory pore 340 - 381 (381), 198 - 210 (192) and 585 - 680 (653), respectively, from anterior extremity. Vulva pre-equatorial, $11.36-12.83(9.11) \mathrm{mm}$ from anterior extremity, at $45-47$ (47) \% of body length. Vulval lips not elevating (Fig. 3I). Vagina muscular, directed posteriorly from vulva. Uterus filled with larvae 447 - 456 (-) long and 24 (24) wide; oesophagus 90 - 93 long (20 - $21 \%$ of body length), tail $117-125$ long (226 $28 \%$ of body length); tail tip of larva bearing several digital projections (Fig. 3G, H). Tail of female rounded, with digit-like projection bearing two small terminal spikes (Figs. 3K, N, 4F, H). Length of entire tail $180-243$ (201); digit-like projection with terminal spikes $45-75$ (57) long and $18-21$ (21) wide; spikes $3-6$ (5) long.

Hosts: Longspine emperor, Lethrinus genivittatus, and slender emperor, Lethrinus variegatus (Lethrinidae, Perciformes).

Site of infection: Intestine.

Locality: New Caledonia (off Nouméa).

Prevalence and intensity: L. genivittatus: 52 fish infected/118 fish examined; 1-5 (mean 3) specimens per fish. L. variegatus: 1 specimen found in 1 fish of 5 examined. Deposition of voucher specimens: Muséum National d'Histoire Naturelle, Paris (JNC 643-648, 653, 658, 659, 1959, 1960, 2022, 2030-2033, 2077, 2228, 2229, 2231, 2234, 2236, 2237, 2239-2247, 2250, 2312, 2317, 2321, 2327, 2507); Institute of Parasitology, BC ASCR, České Budějovice (N-952).

Comments: Nematodes of the present material belong to the morphological group of Procamallanus (Spirocamallanus) species characterized by the presence of wide caudal alae, three pairs of pedunculate preanal papillae and two unequal spicules, that are mostly parasites of marine fishes (Petter, 1979). As pointed out by Petter et al. (1977) and Rigby and Adamson (1997), the shape and structure of the female tail appears to be constant within a species of Procamallanus (Spirocamallanus). To date, six morphologically very similar species of this subgenus possessing two caudal spikes on a digit-like projection in females have been described from marine perciform and atheriniform fishes in the Pacific: $P$. (S.) pereirai Annereaux, 1946, $P$. (S.) monotaxis (Olsen, 1952), P. (S.) istiblenni (Noble, 1966), P. (S.) philippinensis (Velasquez, 1980), P. (S.) guttatusi (Andrade-Salas, Pineda-López et García-Magaña, 1994) and P. (S.) variolae Moravec, Justine et Rigby, 2006 (see Olsen, 1952; Noble, 1966; Velasquez, 1980; Machida
\& Taki, 1985; Andrade-Salas et al., 1994; Moravec et al., 2004, 2006a); of them, P. philippinensis is considered a species inquirenda (see Rigby \& Font, 1997).

Of the above-named valid species, $P$. $(S$.) pereirai, originally described from the atheriniform fish Atherinopsis californiensis Girard (Atherinopsidae) off California, differs from New Caledonian specimens mainly in having a longer right spicule (about 430 vs. 279 - 315), postequatorial (vs. preequatorial) vulva and somewhat different shape (more slender) of the female tail (Annereaux, 1946). Procamallanus (S.) guttatusi from Siganus guttatus (Siganidae) from the Philippines differs by longer gravid females $(30-37$ vs. $25-28 \mathrm{~mm})$ with a distinctly smaller buccal capsule ( 75 - 78 vs. 96 - 105 long), more posterior location of the excretory pore (at level of the glandular oesophagus) and a more slender female tail (Moravec et al., 2004); P. (S.) variolae from Variola albimarginata and Epinephelus fasciatus (both Serranidae) from New Caledonia mainly by the location of deirids at the level of nerve ring, more posterior location of the excretory pore (at level of the glandular oesophagus) and a somewhat longer right spicule $(327-357)$.

Morphologically and biometrically very similar are the species $P$. (S.) monotaxis and $P$. (S.) istiblenni, both originally described from Hawaii from Monotaxis grandoculis Forsskål (Lethrinidae) and Istiblennius zebra (Vaillant et Sauvage) (Blenniidae), respectively. According to Rigby and Adamson (1997) and Rigby and Font (1997, 2001), both these species can be distinguished from each other only by the relative position of the male's second preanal papilla. However, considering the original description by Olsen (1952) and that by Noble (1966), the excretory pore is more posterior (at level of glandular oesophagus vs. at level of the posterior part of muscular oesophagus) and the female tail more slender in $P$. istiblenni than in $P$. monotaxis. Considering that the specimens of the present material are morphologically and biometrically most similar to P. monotaxis, so far the only Procamallanus species reported from a fish belonging to the family Lethrinidae in the Pacific (Rigby \& Adamson, 1997), they are assigned to this species.

In the original description of $P$. (S.) monotaxis (as Spirocamallanus monotaxis), Olsen (1952) reported 16 spiral ridges in the buccal capsule of male and 10 in that of female. According to the redescription of this species by Rigby and Adamson (1997) based on specimens from French Polynesia, there are $10-14$ ridges in the male and $8-10$ in the female. In contrast, both males and females of the present material possess similar numbers of ridges (14 -17 in males and $14-15$ in females), this being apparently within the limits of intraspecific variability.

SEM examination, used for the first time in this species, revealed the presence of a previously unreported narrow circumoral flange. The presence or absence of such flange may be a good differentiating feature between some Procamallanus spp., as has recently been documented for some congeneric species in Africa (Mašová et al., 2011). Only 12 cephalic papillae arranged in three concentric 
circles have now been confirmed by SEM, instead of 14 papillae reported by Rigby and Adamson (1997).

One of the reliable specific features in this morphological group of Procamallanus (Spirocamallanus) spp. is the shape (bifurcate or simple) of deirids, which, however, can be determined reliably only by SEM (González-Solís et al., 2002; Moravec et al., 2004). The first use of this method in $P$. monotaxis shows that deirids in this species are simple, chisel-shaped or with a somewhat narrowed distal end, resembling thus, e.g., those in $P$. guttatusi (see Moravec et al., 2004). As in the foregoing species, first-stage larvae of $P$. monotaxis have been, for the first time, found to be provided with numerous small digit-like processes at the tail tip, previously reported only for larvae of two species of Camallanus (see Moravec \& Justine, 2006). The presence/absence and the character of caudal processes in firststage larvae may be an important taxonomic feature in camallanids (Moravec \& Justine, 2006). Apparently, caudal processes serve the larva to the better attachment by its tail to the bottom, after the larvae are released into the water; then the larvae attract by their movements the copepod intermediate hosts.

In addition to the type host $M$. grandoculis in Hawaii (O1sen 1952), P. monotaxis was reported by Rigby and Adamson (1997) from French Polynesia from the same host species and other ten species of anguilliform, aulopiform, beryciform and perciform fishes, including the lethrinid L. olivaceus Valenciennes. However, the records $P$. monotaxis and $P$. istiblenni may well include each more than a single nematode species (Rigby \& Font, 1997; Moravec et al., 2004, 2006a). Procamallanus monotaxis has not previously been reported from New Caledonian waters and L. genivittatus and L. variegatus represent its first host records. This species seems to be relatively host-specific; in this study, no camallanid was found in 133 specimens of other species of Lethrinus examined, including L. atkinsoni Seale (14 specimens), L. harak (Forsskål) (2), L. lentjan (Lacépède) (7), L. miniatus (Forster) (27), L. nebulosus (Forsskål) (14), L. obsoletus (Forsskål) (3), and L. rubrioperculatus Sato (66).

From the intestine of the congeneric host (L. ravus Carpenter et Randall) off New Caledonia, Moravec et al. (2006a) reported a subgravid female of an unidentified species designated as Procamallanus (Spirocamallanus) sp. 2. Although the structure of its buccal capsule resembles that of $P$. monotaxis, this species distinctly differs from the latter in having a single conical spike on the digit-like caudal projection instead of two in $P$. monotaxis.

\section{Acknowledgements}

Many students in New Caledonia participated in the fishing operations and the parasitological survey. Certain fishes were identified from photographs by Ronald Fricke (Staatliches Museum für Naturkunde, Stuttgart, Germany). Thanks are also due to the staff of the Laboratory of Electron Microscopy of the Institute of Parasitology, Biology
Centre of the ASCR, in České Budějovice for their technical assistance, and Blanka Škoríková from the Department of Helminthology of the same Institute for her help with the preparation of illustrations. This study was partly supported by the research projects of the Institute of Parasitology, BC ASCR (Z60220518 and LC522).

\section{References}

Andrade-Salas, O., Pineda-LóPez, R. F., GarcíAMAGAÑA, L. (1994): Spirocamallanus rebecae sp. $\mathrm{n}$. (Nematoda: Camallanidae) from freshwater fishes in south-eastern Mexico. Folia Parasitol., 41: 259 - 270

AnnereauX, R. F. (1946): A new nematode, Procamallanus pereirai, with a key to the genus. Trans. Am. Microsc. Soc., 65: 299 - 303

ARYA, S. N. (1984): On a new species of Procamallanus Baylis, 1923 from a marine fish of India. Z. Angew. Zool., 71: $459-462$

BAILlON, N. (1991): Otolithométrie en milieu tropical: application à trois espèces du lagon de Nouvelle-Calédonie (Vol. Travaux et Documents Microétités, TDM 113). Bondy, France, Éditions de l'ORSTOM, 290 pp.

Froese, R., Pauly, D. (Eds.) (2010): FishBase. World Wide Web electronic publication, version 05/2010. http://www.fishbase.org

Fusco, A. C., Overstreet, R. M. (1979): Two camallanid nematodes from Red Sea fishes including Procamallanus elatensis sp. nov. from siganids. J. Nat. Hist., 13: 35 - 40

GonZÁlez-Solís, D., Moravec, F., VidAl-Martínez, V. M. (2002): Procamallanus (Spirocamallanus) chetumalensis n. sp. (Nematoda: Camallanidae) from the Mayan sea catfish, Ariopsis assimilis, off the Caribbean coast of Mexico. J. Parasitol., 88: 765 - 768

IVAshkin, V. M., Sobolev, A. A., Khromova, L. A. (1971): Camallanata of animals and man and the diseases caused by them. Essentials of nematodology 22. Moscow, Nauka, 388 pp. (in Russian)

Justine, J.-L. (2010): Parasites of coral feef fish: how much we know? with a bibliography of fish parasites in New Caledonia. Belg. J. Zool., 140 (Suppl.): 155 - 190

LESTER, R. J. G., SEWELl, K. B. (1989): Checklist of parasites from Heron Island, Great Barrier Reef. Austr. J. Zool., 37: $101-128$

MACHIDA, M., TAKI, Y. (1985): A new species of Spirocamallanus (Nematoda, Camallanidae) from rabbitfish in the Philippines. Bull. Nat. Sci. Mus., Ser. A, 11: 53 - 56

MaŠovÁ, Š., BARUŠ, V., Moravec, F., KoubKová, B. (2011): Nematodes of the family Camallanidae from African freshwater fishes. Folia Parasitol., submitted

Moravec, F., Cruz-Lacierda, E. R., NAgAsawa, K. (2004): Two Procamallanus spp. (Nematoda, Camallanidae) from fishes in the Philippines. Acta Parasitol., 49: $309-318$

Moravec, F., Justine, J.-L. (2006): Camallanus cotti (Nematoda: Camallanidae), an introduced parasite of fishes in New Caledonia. Folia Parasitol., 53: 287-296

Moravec, F., Justine, J.-L., Rigby, M. C. (2006a): Some 
camallanid nematodes from marine perciform fishes off New Caledonia. Folia Parasitol., 53: 223 - 239

MoraVEC, F., Justine, J.-L., WÜRTZ, J., TARASCHEWSKI, H., SASAL, P. (2006b): A new species of Procamallanus (Nematoda: Camallanidae) from Pacific eels (Anguilla spp.). J. Parasitol., 92: $130-137$

MYers, B. J., KUNTZ, R. E. (1969): Nematodes of fishes, amphibians, and reptiles taken by U.S. Naval Medical Research Unit No. 2 expedition to North Borneo (Malaysia). J. Fish. Res. Bd. Canada, 26: 793 - 797

NoBle, E. L. (1966): A new camallanid nematode from Hawai'i. Pacific Sci., 20: 360 - 366

OLSEN, L. S. (1952): Some nematodes parasitic in marine fishes. Publ. Inst. Mar. Sci., Univ. Texas, 2: 173 - 215

PARUKHIN, A. M. (1971): Nematodes of fishes of the Red Sea and the Indian Ocean. In: Voprosy ekologii ryb yuzhnykh morey. Biologiya Morya 23. Kiev: Naukova Dumka, pp. 177 - 193 (in Russian)

PARUKHIN, A. M. (1976): Parasitic worms of commercial fishes of the southern seas. Kiev, Naukova Dumka, 183 pp. (in Russian)

Petter, A. J. (1979): Essai de classification de la sousfamille des Procamallaninae (Nematoda, Camallanidae). Bull. Mus. Nat. Hist. Nat., Paris, Sect. A, Zool., No. 1, 991 $-1008$

Petter, A. J., Golvan, Y. J., Tcheprakoff, R. (1977): Nématodes de Poissons de riviere en Guadeloupe. Bull. Mus. Nat. Hist. Nat., Paris, $3^{e}$ sér., no. 428, Zool., 298: $159-171$

RigBy, M. C., Adamson, M. L. (1997): Spirocamallanus species of French Polynesian coral reef fishes. Can. J. Zool., 75: 1270 - 1279

RigBy, M. C., AdAMson, M. L., DeArdorfF, T. L. (1998): Camallanus carangis Olsen, 1954 (Nematoda: Camallanidae) reported from French Polynesia and Hawai'i with a redescription of the species. J. Parasitol., 84: 158 - 162

RigBY, M. C., FONT, W. F. (1997): Redescription and range extension of Spirocamallanus istiblenni Noble, 1966 (Nematoda: Camallanidae) from coral reef fishes in the Pacific. J. Helminthol. Soc. Wash., 64: 227 - 233
RigBY, M. C., FonT, W. F. (2001): Statistical reanalysis of the distinction between Spirocamallanus istiblenni and $S$. monotaxis (Nematoda: Camallanidae). J. Parasitol., 87: $1210-1213$

Rigby, M. C., Holmes, J. C., CribB, T. H., Morand, S. (1997): Patterns of species diversity in the gastrointestinal helminths of a coral reef fish, Epinephelus merra (Serranidae), from French Polynesia and the South Pacific Ocean. Can. J. Zool., 75: $1818-1827$

Rigby, M. C., Lo, M. C., CRiBb, T., Euzet, L., FAlieX, E., Galzin, R., Holmes, J., Morand, S. (1999): Checklist of the parasites of coral reef fishes from French Polynesia, with considerations on their potential role in these fish communities. Cybium, 23: $273-284$

SigurA, A., Justine, J.-L. (2008): Monogeneans of the speckled blue grouper, Epinephelus cyanopodus (Perciformes, Serranidae), from off New Caledonia, with a description of four new species of Pseudorhabdosynochus and one new species of Laticola (Monogenea: Diplectanidae), and evidence of monogenean faunal changes according to the size of fish. Zootaxa, 1695: $1-44$

SMALES, L. R. (2000): Nematodes from fish from the Keppel Islands, Keppel Bay, Queensland. Mem. Queensl. Mus., 45: 384

Teitelbaum, A., Prior, T., Legarrec, F., OengPePa, C., MESIA, P. (2008): Rabbitfish: a candidate for aquaculture in the Pacific? SPC Fisheries Newsletters, 127 (October/December 2008): $40-44$

VelasqueZ, C. C. (1980): Camallanidae (Nematoda) from Philippine fishes. Fish. Res. J. Philipp., 5: 53 - 62

YAMAGUTI, S. (1935): Studies on the helminth fauna of Japan. Part 9. Nematodes of fishes, 1. Jpn. J. Zool., 6: 337 $-386$

YAMAGUTI, S. (1941): Studies on the helminth fauna of Japan. Part 33. Nematodes of fishes, II. Jpn. J. Zool., 9: 343 - 395, Plts. IV - VI

YAMAGUTI, S. (1955): Parasitic worms mainly from Celebes. Part 9. Nematodes of fishes. Acta Med. Okayama, 9: $122-133$, Plts. I, II

ACCEPTED DeCEMBER 6, 2010 